\title{
A MODEL OF THE CHROMOSPHERE FROM RADIO AND OPTICAL DATA
}

\author{
R. G. ATHAY \\ High Altitude Observatory, Boulder, Colorado, U.S.A.
}

\section{INTRODUCTION}

During the past few years various chromospheric models based on optical and ratio data have appeared in the literature. They exhibit an overwhelming tendency to recognize the chromosphere as a heterogeneous medium departing markedly from spherical symmetry. Beyond this general trend, however, their similarity all but vanishes. Perhaps the reason for this dissimilarity lies in the complexity of nonspherically symmetric models. The simpler ones, based on the assumption of spherical symmetry, however, show an equal diversity. It seems, therefore, that the diversity must be attributed to a lack of basic understanding of chromospheric phenomena.

Once we abandon the relatively simple models of a spherically symmetric chromosphere, the next simplest alternative is a two-component model consisting of "hot"- and " cold "-volume elements, in each of which temperature and density vary in the radial direction only. Even in such a model, however, five parameters (temperature and density in each component and the relative proportions of the two components) must be evaluated at each height where the thermodynamic state of the atmosphere is specified. Thus, at each height where the model is specified, five independent observational data are needed. In general, the available data are not adequate to completely specify such a model. The problem is further complicated by the realization that in using observational data we are justified in evaluating only the minimum number of parameters that are required to represent the data. Indeed, if we are to have confidence in a model it must be capable of explaining more data than are required to specify its parameters.

The purpose of this paper is to demonstrate that the radio data can be represented by a fairly simple two-component model whose main features are derived from optical data. The basic initial model contains four unspecified parameters. These four parameters are evaluated from central disk brightness temperatures at wavelengths of $4 \mathrm{~mm}, 8.6 \mathrm{~mm}, 3 \mathrm{~cm}, 10 \mathrm{~cm}$, and $21 \mathrm{~cm}$, and center-to-limb brightness temperatures at $8.6 \mathrm{~mm}$.

\section{RADIO DATA}

Central brightness temperatures for the solar disk are available at several wavelengths in the radio spectrum, but only the shortest wavelengths are of 
interest in connection with the chromosphere. The data used in this analysis are summarized in Table I.

TABLE I

\section{Central Brightness Temperatures}

\begin{tabular}{|c|c|c|c|}
\hline$\lambda$ & $\begin{array}{c}\text { Brightness } \\
\text { Temperature }\end{array}$ & Method & Reference \\
\hline $4 \mathrm{~mm}$ & $7,000^{\circ} \mathrm{K}$ & 1954 eclipse & [1] \\
\hline $8.6 \mathrm{~mm}$ & 8,500 & 1954 eclipse & [1] \\
\hline \multirow[t]{3}{*}{$3 \mathrm{~cm}$} & 19,000 & 1954 eclipse & [2] \\
\hline & 16,000 & 1952 eclipse & [3] \\
\hline & 17,400 & noneclipse & [4] \\
\hline \multirow[t]{2}{*}{$10 \mathrm{~cm}$} & 23,000 & 1954 eclipse & [5] \\
\hline & & noneclipse & [6] \\
\hline $21 \mathrm{~cm}$ & 47,000 & noneclipse & [7] \\
\hline
\end{tabular}

Central brightness temperatures at different wavelengths effectively map the radial temperature structure of the middle and upper chromosphere. However, they tell us nothing about the nonspherical properties of the chromosphere. For this, we must turn to observations of center-to-limb variations in brightness temperature. We utilize the data at millimeter wavelengths in which the emission is exclusively chromospheric. Fig. 1 shows the limiting values of brightness temperature across the solar disk at an $8.6 \cdot \mathrm{mm}$ wavelength [1] as derived from 1954 eclipse data (dashed lines). We shall use these data in conjunction with the central brightness temperatures. The most striking feature of the center-to-limb data is the apparent constancy of brightness temperature out to $R / R_{\odot} \sim 0.98$. Hagen [8] and Coates [1] have pointed out that models derived from central brightness temperatures alone predict a monotonic increase in brightness temperature from center-to-limb that should be readily observed for $R / R_{\odot} \geq 0.8$. They further point out that the absence of limb-brightening out to $R / R_{\odot} \sim 0.98$ can be logically explained only by resorting to models

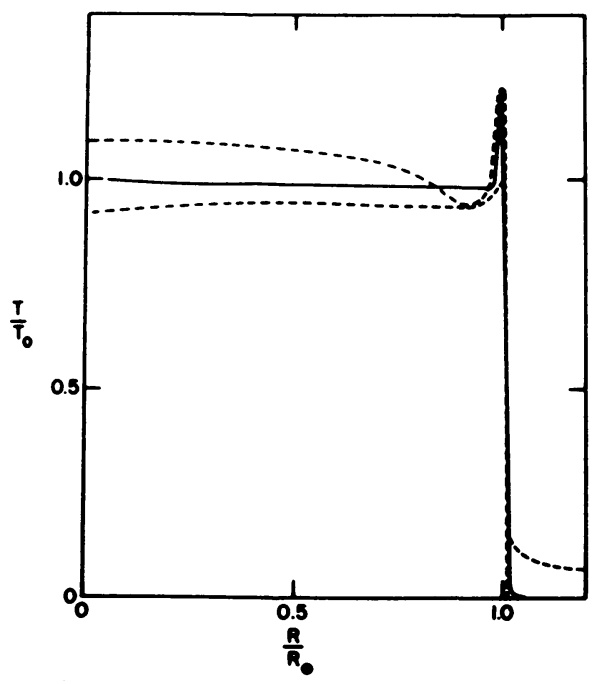

FIG. 1. Center-to-limb variations in brightness temperature at $8.6 \mathrm{~mm}$. that depart from spherical symmetry.

In using the radio data to evaluate parameters of a model, we must determine the number of parameters required to represent the data. A curve lying within the limits of center-to-limb brightness temperatures in Fig. 1 can be 
specified by four points. These four points together with the data in Table I constitute eight observations. It is unlikely, however, that these eight observations are all independent, since center-to-limb observations are in some ways equivalent to central-brightness temperatures at different wavelengths. Our model, therefore, must contain less than eight adjustable parameters. Since a realistic model cannot be specified with so few parameters, we start with a model derived from optical data.

\section{THE INITIAL MODEL}

As an initial model, we adopt the model derived by Athay and Menzel [9] and later modified by Athay and Thomas [10]. Fig. 2 represents a schematic illustration of this model. Along any given radial direction the kinetic temperature, $T_{e}$, increases outward in three discrete, unique steps [11]. The cold components are distinguished from the hot components by the heights at which these steps occur.

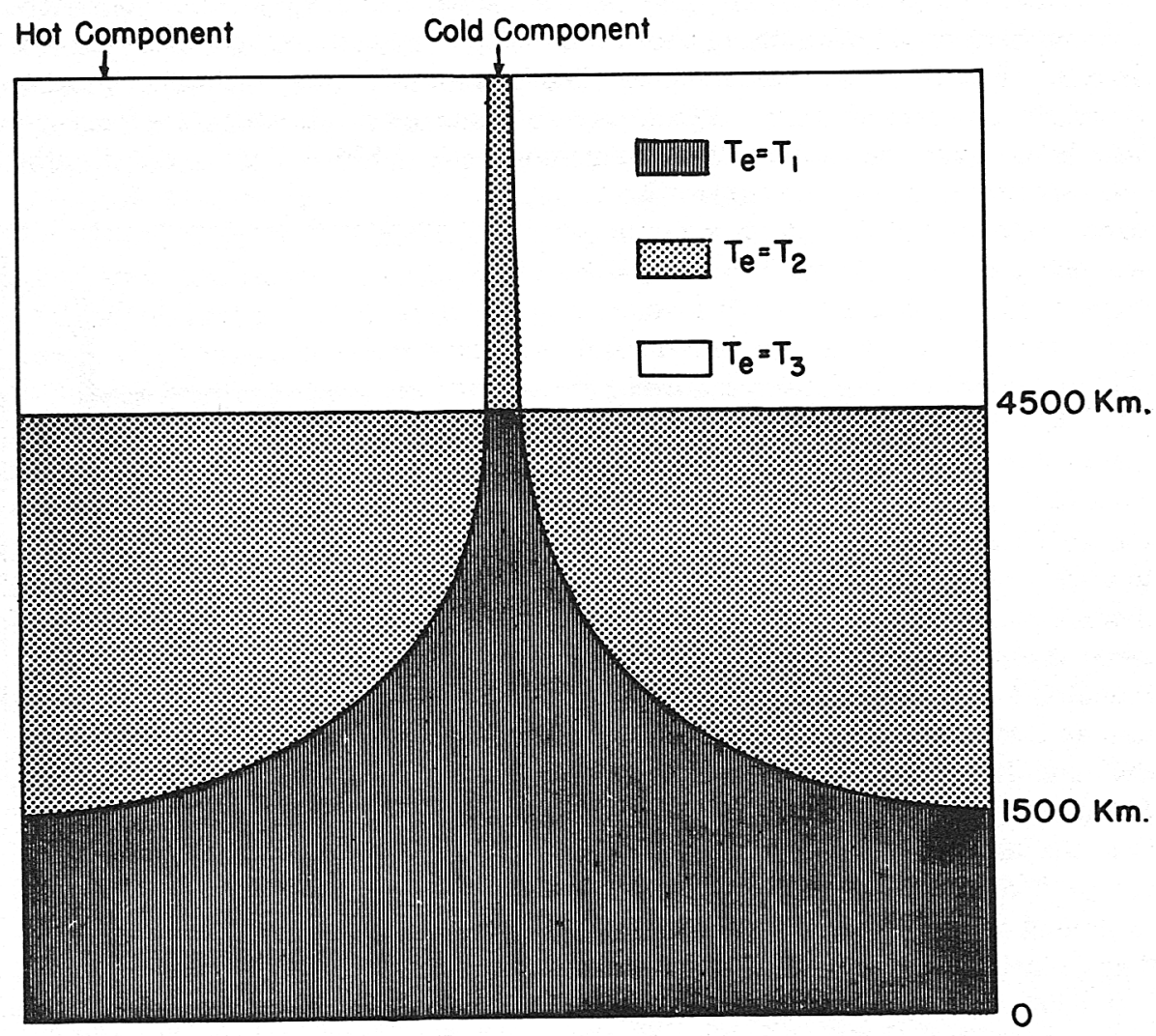

Fig. 2. A schematic model of the chromosphere derived from optical data. 
In the following, we define :

$T_{i}=$ kinetic temperature of temperature step $i$;

$\tau_{i}=$ optical thickness of temperature step $i$;

$n_{\imath}=$ electron density of temperature step $i$;

$a_{i}=$ fractional area of the cold component of the atmosphere at temperature step $i$.

In the model $T_{i}$ remains constant with height, but $\tau_{i}, n_{i}$, and $a_{i}$ vary with height. To a degree of approximation sufficient for the present purposes, $n_{i}$ varies exponentially with height. The two components of the atmosphere are at temperature steps 1 and 2 below $4500 \mathrm{~km}$ and at steps 2 and 3 above $4500 \mathrm{~km}$. We identify the cold components above $4500 \mathrm{~km}$ with spicules.

Brightness temperatures at radio wavelengths depend upon $T_{i}, \tau_{i}$, and $a_{i}$; and $\tau_{i}$, in turn, depends upon $T_{i}$ and $n_{i}$. Since we require an initial model with less than eight parameters, we choose as many parameters from the optical model as seem well determined. From the numerical values of these parameters, we arrive at the following initial model :

TABLE II

$$
\begin{aligned}
& 0-1500 \mathrm{~km} \\
& T_{1}=6500^{\circ} \mathrm{K} \\
& n_{1} \sim 10^{11} \\
& \dot{a}_{1}=1
\end{aligned}
$$$$
1500-4500 \mathrm{~km}
$$$$
4500 \mathrm{~km}
$$$$
T_{1}=6500^{\circ} \mathrm{K}
$$$$
n_{1} \sim 10^{11}
$$$$
a_{1}=\text { unknown }
$$$$
T_{2}=\text { unknown }
$$$$
n_{2} \sim 10^{11}
$$$$
a_{2}=\text { projected area }
$$$$
T_{2}=\text { unknown }
$$$$
\text { of spicules }
$$$$
n_{2}=n_{2,0} e^{-\beta_{2} h}
$$$$
T_{3}=10^{6}
$$$$
\beta_{2}=10^{-8} \mathrm{~cm}^{-1}
$$$$
n_{3}=n_{3,0} e^{-\beta_{3} h}
$$$$
\beta_{2}=2 \times 10^{-10} \mathrm{~cm}^{-1}
$$$$
n_{2,0}=\text { unknown }
$$$$
n_{3,0}=\text { unknown }
$$

The initial model contains three unknowns, $T_{2}, n_{2,0}$, and $n_{3,0}$, in addition to $a_{1}$ in the layers from 1500 to $4500 \mathrm{~km}$. In order to further simplify the model, we assume that the cold elements at temperature $T_{1}$ between 1500 and $4500 \mathrm{~km}$ are observed at an average height of $3000 \mathrm{~km}$, and that they have a mean diameter of $1000 \mathrm{~km}$. We then have only the number, $q$, 'of these elements to determine, which reduces the number of unknowns in the model to four.

The electron density. in the first step in the temperature curve and in the spicules is of the order $10^{11}$, which results in large optical thickness at wavelengths of $4 \mathrm{~mm}$ or greater. Thus, we cannot specify the electron density in these regions from existing radio data.

\section{THE FINAL MODEL}

The projected area of spicules extending above $4500 \mathrm{~km}$ may be obtained from counts of spicules at the limb [12]. Spicule numbers corrected for projection and obscuration effects are shown in Table III. The mean spicule 
diameter is about $700 \mathrm{~km}$. Hence, at the center of the disk we obtain $a_{2} \sim$ 0.004 for spicules extending above $4500 \mathrm{~km}$.

TABLE III

SPICULE NUMBERS

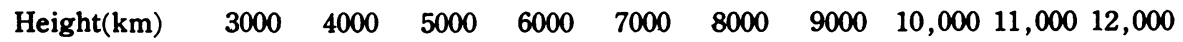

Number of spicules on sun $\times 10^{-4}$

$\begin{array}{llllllllll}9.3 & 6.8 & 5.5 & 4.2 & 2.9 & 1.8 & 1.1 & .55 & .32 & .15\end{array}$

Preliminary calculations show that $a_{1}$ at $3000 \mathrm{~km}$ is much greater than $a_{2}$ at $4500 \mathrm{~km}$. Hence, we may use $a_{1}$ at $3000 \mathrm{~km}$ to approximate the area of cold components above $1500 \mathrm{~km}$. In our simplified model, the brightness temperature at angle $\theta$ from the center of the disk and wavelength $\lambda$ is given by the sum of the contributions from hot and cold elements; viz. :

$$
\begin{aligned}
T(\theta, \lambda)= & T_{1}\left[1-a_{1}(\theta, q)\right] \exp -\left[\tau_{2}(\theta, \lambda)+\tau_{3}(\theta, \lambda)\right] \\
& +T_{1} a_{1}(\theta, q) \exp -\left[\frac{1}{20} \tau_{2}(\theta, \lambda)+\tau_{3}(\theta, \lambda)\right] \\
& +T_{2}\left[1-a_{1}(\theta, q)\right]\left\{\exp -\left[\tau_{3}(\theta, \lambda)\right]-\exp -\left[\tau_{2}-(\theta, \lambda)\right]\right\} \\
& +T_{2} a_{1}(\theta, q)\left\{\exp -\left[\tau_{3}(\theta, \lambda)\right]-\exp -\left[\frac{1}{20} \tau_{2}(\theta, \lambda)\right]\right\} \\
& +T_{2} a_{2}(\theta, q) \exp -\left[\tau_{3}(\theta, \lambda)\right]+T_{3}\left\{1-\exp -\left[\tau_{3}(\theta, \lambda)\right]\right\}
\end{aligned}
$$

The factor $1 / 20$ multiplying $\tau_{2}(\theta, \lambda)$ in some of the terms in equation (1) arises from the decrease in $\tau_{2}$ between 1500 and $3000 \mathrm{~km}$, which amounts to $e^{-3}$.

The line-of-sight optical depth at height $h$ above the base of an isothermal layer is

where

$$
\tau_{i}(\theta, \lambda)=\alpha_{i}(\lambda) \int_{h}^{\infty} e^{-2 \beta i n} d y
$$

$$
\alpha_{i}(\lambda)=2.5 \times 10^{-23} \lambda^{2} n_{i, 0}^{2} T_{i}^{-3 / 2}\left(3.23+\log T_{i}-\frac{1}{3} \log n_{i}\right)
$$

[13] and $y$ is the coordinate in the line of sight. To a good approximation

$$
h=\frac{y^{2}}{2 R_{\odot}}+y \cos \theta \text {. }
$$

In order to determine $a_{1}(\theta, q)$ and $a_{2}(\theta, q)$, we assume a random distribution of cold elements, in which case the Poisson relation gives

$$
\alpha_{i}(\theta, q)=1-\exp -\left[A_{i}(\theta, q)\right] \text {. }
$$

$A_{i}(\theta, q)$ is the total surface area of cold elements projected against a unit area of the solar disk, ignoring the fact that one cold element may lie in front of another. Thus if $q$ is large, when we look at the cold elements 
near the edge of the disk $A_{\mathfrak{i}}(\theta, q)$ may be considerably greater than unity. However, $a_{i}(\theta, q)$ is always less than unity.

Solutions of the foregoing equations for $T_{2}, n_{2,0}, n_{3,0}$, and $q$ are readily obtained by iterative methods. As is to be expected with a simplified model containing fewer parameters than the number of observational data to be fitted, the data cannot all be fitted precisely, and there is a choice to be made about which data should be fitted best. Since the chromospheric model is most sensitive to data for $\lambda \leq 3 \mathrm{~cm}$, we adopt the model that best represents these data.

The two density parameters $n_{2,0}$ and $n_{3,0}$ and the number of cold elements, $q$, are well determined by the model since they enter the equations through exponential terms. $T_{2}$, however, is not as well determined since it enters the equations primarily as a linear term. Acceptable representations of the data can be obtained for $T_{2}$ in the range $20,000-30,000^{\circ} \mathrm{K}$.

The adopted model is given in Table IV and the computed central brightness temperatures are given in Table $\mathrm{V}$. The center to limb variations in brightness temperature at $8.6 \mathrm{~mm}$ are shown in Fig. 1 as the solid curve.

TABLE IV

\begin{tabular}{|c|c|c|c|c|c|}
\hline \multicolumn{6}{|c|}{ ADOPTED MODEL } \\
\hline & & $n_{2,0}$ & $n_{3,0}$ & \multicolumn{2}{|c|}{$q$} \\
\hline & & $1.4 \times 10^{10}$ & $3 \times 10^{8}$ & \multicolumn{2}{|c|}{$3.4 \times 10^{6}$} \\
\hline \multicolumn{6}{|c|}{ TABLE $\mathrm{V}$} \\
\hline \multicolumn{6}{|c|}{ COMPUTED $T(0, \lambda)$} \\
\hline$\lambda$ & $4 \mathrm{~mm}$ & $8.6 \mathrm{~mm}$ & $3 \mathrm{~cm}$ & $10 \mathrm{~cm}$ & $21 \mathrm{~cm}$ \\
\hline$T(0, \lambda)$ & 7100 & 8500 & 16,000 & 26,000 & 40,000 \\
\hline
\end{tabular}

The adopted model differs basically from Coates' model [1] in the temperature structure and in the number of spicules. The agreement between computed and observed central disk and center-to-limb brightness temperatures is rather striking, especially since we have used a model with only four free parameters. Furthermore, the model differs significantly from the model derived solely from optical data in the parameters $n_{2,0}$ and $n_{3,0}$ only. The remaining two parameters are both highly consistent with the optical model.

Both $n_{2,0}$ and $n_{3,0}$ are lower in the radio model than in the optical model by about a factor of ten. This discrepancy is not as serious as it appears to be. There is good evidence from the optical data that the absolute intensities are too high by a factor of two to three [14], which results in a similar error in $n_{\mathbf{2}, 0}$ and $n_{3,0}$. In addition, alternative representations of the optical data in analytical form permit another factor of two reduction in $n_{2,0}$ and $n_{3,0}$. Admittedly, it seems difficult to fully reconcile the two models. Nevertheless, the discrepancies in electron density are not completely outside the possible errors in the two models. Part of the difficulty may arise from an oversimplification of the models. 
With the value of $q$ given in Table IV, about 50 per cent of the solar surface is covered by cold elements at $3000 \mathrm{~km}$. The optical model gives a somewhat lower value, which, however, increases rapidly downward so that 50 per cent of the surface is covered at about $2000 \mathrm{~km}$. On the other hand, the spicule counts in Table III give only about 0.4 per cent of the solar surface covered by spicules at $3000 \mathrm{~km}$. It appears, therefore, that we should not identify the cold elements of the low chromosphere with spicules. The high percentage of the solar surface covered by cold elements is suggestive of the fine mottling observed in $\mathrm{H} \alpha$ spectroheliograms.

Finally, we note that the limits on $T_{2}$ of 20,000 to $30,000{ }^{\circ} \mathrm{K}$ are very nearly the same as the limits derived from optical data. We should note, however, that the spicules extending above $4500 \mathrm{~km}$ contribute significantly to the observed brightness temperature at the extreme limb only. Thus, if we permit a change in $a_{2}(\theta, q)$ of a factor of two, we can permit a corresponding inverse change in $T_{2}$ in the spicules. Recent studies of line profiles in spicules indicate a kinetic temperature considerably above $25,000^{\circ} \mathrm{K}$ [15], which suggests that we have overestimated $a_{2}(\theta, q)$ at the limb owing to an overestimate of the mean spicule diameter.

The most desirable radio data for obtaining improved models of the chromosphere are center-to-limb observations of brightness temperature at $\mathrm{mm}$ and $\mathrm{cm}$ wavelengths with particular emphasis on the region near the limb.

The work reported in this paper was supported by the Air Force Cambridge Research Center, Geophysical Research Directorate, through contract AF 19 (604)-2140 with the High Altitude Observatory.

\section{REFERENCES}

[1] Coates, R. J. Thesis, Johns Hopkins University, 1957.

[2] Hachenberg, O., Fürstenberg, F., and Prinzler, H. Z. Ap. 39, 232, 1956.

[3] Blum, E. J., Denisse, J. F., and Steinberg, J. L. Ann. Astrophys. 15, 184, 1952.

[4] Minnett, H. C., and Labrum, N. R. Aust. J. Sci. Res. A 3, 60, 1950.

[5] Haddock, F. T. Radio Astronomy (I.A.U. Symposium No. 4, 1955). Cambridge, England, 1957, p. 273.

[6] Covington, A. E., and Broten, N. W. Ap. J., 119, 569, 1954.

[7] Christiansen, W. N., and Warburton, J. A. Aust. J. Phy8. 8, 474, 1955.

[8] Hagen, J. P. J. Geophys. Res. 59, 158, 1954.

[9] Athay, R. G., and Menzel, D. H. Ap. J. 123, 285, 1956.

[10] Athay, R. G., and Thomas, R. N. Monograph on solar chromosphere (in preparation), 1958.

[11] Athay, R. G., and Thomas, R. N. Ap. J. 123, 299, 1956.

[12] Athay, R. G. In press, 1958.

[13] Martyn, D. F. Proc. Roy. Soc. A 193, 44, 1948.

[14] Athay, R. G., Menzel, D. H., and Orrall, F. Q. Smithson. Contr. Astro. 2, 35, 1957.

[15] Athay, R. G. Ann. Astrophys. In press, 1958. 